und der Yermcidung unü̈iger Eunengungen, hat er dagegen überzeugend betont. Geradi $1 \mathrm{~m}$ der letzteren willen mochto ich aber die von mir vorgeschlagene, "Inuenschiene" mit tinem kurzen Thoraxteil der von Burchers vorgeschlagenen "Fxtensionstriangel“ mit langem Thoraxteil vorzichon. Jiesen hatte ich anfänglich auch angewandt, bin aher rasch duvon abgokonmen, weil er die Rumpf boweglichkeit unnötig stort, ohule ene bessere Fixation des gebrochenen Gliedes zu gewährleisten. Schr raten möehte ich außerdem zal der voll mir angewandten dritten Biegung, der leichten Dorsalflexion der Hand. Sie entsprieht der natürlichen Durchschuittshaltung, vermeidet Handgelenksversteifung und orleichtert nebenbei das Anbandagieren der Schiene.

Zur Klarstellung der Priorität bemorkë ich, daB jeh meine fertige Arbcit um 9. April 1915 meiner vorgesetzten Behörle und am 4 Mai 1915 zur Voroffentlichung in (ler I). $\mathrm{m}$. W. eingereicht habc. ${ }^{1}$ )

\title{
Extensionstriangel oder Innenschiene?
}

Von Dr. L. Engelbarit,

z. Z. Assistenzarzt bei cince Sanitat,konulagnic.

Das bekannte Gexetz vou der louplizitian der Zufälle hat genollt,

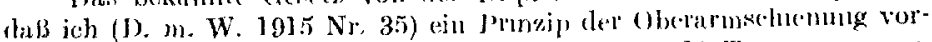
reblug, welehes der von Herru Kollegen Bolechers 16 Tage zuvor ver-

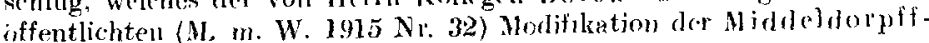
schen 'Triangel gleichfalls zugruudeliegt.

Win Vergleich unserer Arbeiten zeigt, claks wir auf gheichem Wege uncl aus gleichen Erwägungen anf gan\% ahuliehe lösungen (ines alten Problens gekommen sind. Vollkommen stimuen wir nicht übereis. Während teh sozusagen nur due mechanisehen Prinzipien iu aller Kiurz: präzisiert habe, hat Herr Kollege Borehers ansführlich anf die Literatur Beyug genommen und hat die bisher den Oberarmbriichen gegellïber bestehonde, Verlegenheit". dle großen Vorteile des neuen V(rïber bestehende ,Verlegenheit". dle großen Vorteile des neuen VerVor allom sheinen unir aber ans seince Darstellung zwei wissensehaftlich wesentliche Moncute nicht klar genug hervorautreten, nämlich 1 . daß \& s wesentiche Momente nicht ist, eiler dislocatio ad longitudinem durch Gug von der AuBenscite her (Aarauf läuft ja, wie ich gezeigt habe, dic Außenschienung und läuft aueh dor ïbliehe große Gipsverband hinaus) vorzubeugen, weun kelartiger bruck an der Innenseite auwendbar int. vorzubengen, wein kellartiger Druck an der Inmenseite anwendbar int. 2. daß außenscitige Verbandsstiitzung speziell an Oberarum deshalb
quoad longitudinem wertlos ist, weil die AuBenlïngsseite eino inkonstant: Größe ist im Gegensat\% zur Innculaugsseite.

Das dritte, dic Bedeuturng der nommilen Durehsehnitshaltung

1. Incrischen hat sich der Zustand soweit gebessert, dals am 15. Nov sich in ler ersten Haruportion nur noch vereinzelte dimnc Fuclen funden, 2 und 3 ist klar Ich habe Einspritanngen mit zon Falen forder Methylenblanlosung machen lassen, woHulfte verdiunter labere Hiterest binnen 24 Stunden verschwand. rauf der noch gebliebene terest debrameh in Folde gerignet! 